\title{
Open Source Research: A Proposed Method to Study Peripartum Myocardial Infarction
}

\author{
Philip D Houck* \\ Department of Medicine, Division of Cardiology, Texas A\&M Health Science Center, Baylor Scott \& White Health, TX 76508.
}

\begin{abstract}
Received: July 23, 2016; Accepted: July 30, 2016; Published: August 15, 2016
*Corresponding author: Philip David Houck, Texas A\&M Health Science Center, Cardiology Division, 1H, Baylor Scott \& White Health, 2401 South 31 st Street, Temple, Texas 76508, Tel: 254-724-6782;Fax: 254-724-2661;E-mail: phouck@sw.org
\end{abstract}

\begin{abstract}
Rare diseases such as peripartum myocardial infarction are difficult to study using double blind randomized controlled clinical trials. There have been no new advancements in the conduct of clinical trials since 1947. As a result, rare diseases have not been effectively studied despite a gradual gain in knowledge of potential mechanisms. This paper suggests a method of study that can be applied to rare disorders by an older method suggested by James Lind - the controlled clinical trial. Open source models similarly developed by software engineers will allow free access to multiple protocols and allows the physician to choose the protocol that seems most promising. Free access of clinical data will be available to help the physician decide which protocol is successful. Results will be updated continuously by a biostatistician compared with historical control and other competing protocols.

Open Source - as a philosophy that promotes a universal access via free license to a product's design or blueprint, and b) universal redistribution of that design or blueprint, including subsequent improvements to it by anyone.

Open-source model includes the concept of concurrent, yet different agendas and differing approaches in production.

The components of Open source research consist of a WEB site, governing body, data partner, biostatistician, tissue, genetic, proteomic specimen analysis, and funding. The site will be a center for literature review, protocol and protocol rational, consent forms, data collection center, case material, data analysis, and provide on line future publications generated by this open data source. Open source research is different from a registry in that the protocols are based on a biologic model and selected by the physician. Open source allows multiple models to be tested. A successful protocol suggests the model of disease is correct. Results are recorded similarly to a registry and can be used to assess the real time efficacy each protocol used. This method allows an iterative adjustment of treatments until the most effective therapy is found.
\end{abstract}

Keywords: Research; Open Source; Pregnancy Associated Myocardial Infarction;

\section{Background}

Pregnancy associated myocardial infarction is considered rare with a reported incidence of 1 in 16,000 births. Mortality from this event is estimated to be 20 percent $[1,2]$. Peripartum cardiomyopathy has a more frequent occurrence of 1 in 3,200 births. Mortality rates have been reported between 0 and $19 \%$ and cardiac transplant necessary in $6 \%$ to $11 \%$. Fortunately, $50 \%$ of the myopathy patients will spontaneously improve $[3,4]$. The etiology of these two disease states is not clearly determined and may overlap. Postpartum myocardial infarction is specific for vascular disease and can be quantified by a coronary angiogram. Whereas, postpartum cardiomyopathy may have multiple etiologies including hypertension, salt excess and immune causes all of which reduce left ventricular function as measured by echocardiogram. A growing body of evidence suggests the immune system is responsible for peripartum myocardial infarction and in some cases of peripartum cardiomyopathy. The immune system is stimulated by paternal fetal antigens that cross react with maternal tissues $[5,6]$. These rare events are further complicated by spontaneous recovery or devastating poor outcomes. Therapies must be designed that do no harm to the spontaneous recovery group. Clinical trials using randomized double blind placebo control have not been applied to this small cohort of patients due to these complexities, resulting in very little clinical movement [7]. The purpose of this paper is to propose a method of study that can be applied to peripartum myocardial infarction and to other rare disease processes for which a randomized clinical trial is impractical. The protocol favored by the author is modification of the immune system while inhibiting the coagulation cascade. There is growing evidence that coronary dissections have better outcome by conservative management. This evidence is generally reported as case reports or case series. This decision is usually in the hands of the interventionist and the appearance of the coronary angiogram. Open source research allows for these decisions. Recording the results of conservative or interventional can give support to one method or another. When no intervention is possible, the immune modifying protocol can be selected. 
A brief review of medical intervention and ethics will be used to introduce this topic, making a case to retreat to the methods of James Lind with the informational power of open resource format and the WEB.

\section{Evolution of Medical Research}

Table 1 is a brief time line of the evolution of medical research [8]. James Lind is credited for the first controlled clinical trial. An excerpt from his diary explains his study. His suspicion was that nutritional supplements could cure the scurvy. He did not know of vitamin $\mathrm{C}$ and had no knowledge of its pathways.

"May 1747, I selected twelve patients in the scurvy...

Two were ordered each a quart of cyder a day.

Two others took twenty-five drops of elixir vitriol three times a day

Two others took two spoonfuls of vinegar three times a day

Two of the worst patients were put on a course of sea-water

Two others had each two oranges and one lemon given them every day

The two remaining patients, took an electary recommended by a hospital surgeon...

\section{Results}

...the most sudden and visible good effects were perceived from the use of oranges and lemons; one of those who had taken them, being at the end of six days fit for duty ... The other was the best recovered of any in his condition; and ... was appointed to attend the rest of the sick. Next to the oranges, I thought the cyder had the best effects" [9].

Table I: The Evolution of Medical Research

562 BC - 1537: Pre-James Lind Era - The Bible Book of Daniel King Nebuchadnezzar

Result: Vegetarians appeared better nourished than the meat-eaters

1547: Ambroise Pare - Boiling oil in short supply for battle wounds Result: Digestive made of yolks of eggs, oil of roses and turpentine inflicted less pain

1747: James Lind and Scurvy Trial - Controlled clinical trial Result: Oranges and lemons improved scurvy, but was expensive 1800: Arrival of Placebo - 1863 United States physician Austin Flint planned the first clinical study comparing a dummy remedy to an active treatment. He treated 13 patients suffering from rheumatism Result: The favorable progress of the cases was such as to secure for the remedy, generally the entire confidence of the patients

1943: The First Double Blind Controlled Trial - Patulin for Common Cold - the first double blind comparative trial with concurrent controls non-randomized

Result: Common cold was not cured

\section{6: First Randomized Curative Trial - The first randomized} control trial of streptomycin in pulmonary tuberculosis was carried out in 1946 by MRC of the UK

Result: Streptomycin and bed-rest ( $\mathrm{S}$ case) was better than bed-rest alone (C case). Note: Streptomycin was in short supply otherwise this study would not have been performed
This trial with only 12 patients was successful despite an overall trial failure with only four of the patients having a suspected good outcome. Applying statistics to this trial would not have been possible, but medical knowledge advanced.

The last breakthrough in medical studies is attributed to Great Britain and the Medical Research Council of 1946. The trial was the first randomized controlled trial of streptomycin in pulmonary tuberculosis. One of the factors that helped launch this new medical research approach was the shortage of streptomycin. The trial was accepted because it gave patients an even chance to get the drug when there was not enough to go around. Randomization was a new concept championed by Sir Leonard Erskine Hill. "I deliberately left out the words 'randomization' and 'random sampling numbers' at that time, because I was trying to persuade doctors to come into controlled trials in the very simplest form and I might have scared them off “[10].

\section{The Doctor Conundrum}

Hill was correct. Doctors scare easily. If there is no preconceptual bias in a new therapy, the doctors will consider the pretest likelihood to be $50 \%$. Doctors will enroll their patients in randomized trials using the ethics - The good of the population exceeds the potential harm to the patient. On the other hand, if the therapy is judged to be favorable with a pretest likelihood of 51 $-100 \%$, then doctors will not enroll their patients in a randomized clinical trial citing the ethics - The good of the patient exceeds potential harm to the population. Two sins can be implicated omission and commission. Omission is when the doctor should act, but does not. Commission is when the doctor acts, but should not. Ethics and medical research/medical decision-making cannot be separated. These issues are even more compounded when the patient is pregnant, since there are now two lives to consider in any treatment decisions. In the case of pregnancy, a spillover to guidelines can occur. One example is ILCOR Advisory Statement Therapeutic Hypothermia after Cardiac Arrest. An Advisory Statement by the Advanced Life Support Task Force of the International Liaison Committee on Resuscitation "Until further data are available, therapeutic hypothermia should not be used for patients with severe cardiogenic shock or life-threatening arrhythmias, pregnant patients, or patients with primary coagulopathy" [11]. The above statement is a potential example of the sin of omission. Doctors who ignore the guideline and treat the pregnant patient obligate a sin of commission. The data implicating hypothermic harm to the fetus comes from information obtained from pregnant women who required hypothermia during their cardiovascular procedure. Doctors have to decide between the omission sin to the mother and commission sin to the fetus based on little data. Table 2 represents the evolution of ethical and regulatory framework in medical research [12]. The doctor is the final authority in medical-decision making. Research trials have to respect this power since with the authority there is responsibility. Open source research can meet this need and provide useful medical information by retreating to the methods of James Lind and the simple clinical trial to test a hypothesis or model of disease. 


\section{Methods - Open Source Research}

Wikipedia provides a suitable definition of Open Source - as a philosophy that promotes a universal access via free license to a product's design or blueprint, and b) universal redistribution of that design or blueprint, including subsequent improvements to it by anyone [13]. Open-source model includes the concept of concurrent yet different agendas and differing approaches in production.

Open Source research implies that a number of different protocols are available to solve a clinical problem. Access is open to all registered physicians. The source will provide clinical science information, research protocols, consent forms, and be designated as data collection center for all of the data entered by the approved protocols. A governing committee will oversee the protocols submitted for scientific merit and safety. A biostatistician will oversee the results of each protocol. The treating physician will choose a protocol from this site and obtain local approval from their Investigational Review Board (IRB). This task will be fast tracked by the IRB's knowledge that governing committee approved the protocol before being made available as an open source protocol. The governing committee will routinely send to all local IRB's the protocols that have been approved specifically for the treatment of peripartum myocardial infarction. The local committee will act on these protocols as they deem fit and the protocols will be active for recruitment when a patient presents in the future. Each site will be locally managed. The physician or his designee will be responsible for collecting data and entering the data into the Open Source site. These data are depersonalized for the privacy of the patient, but is accessible to all physicians. Outcomes are immediately available and can serve as an aid in the physicians' choice of which protocol to choose for their patient. The Web site and data are part of a quality initiative to determine the safest and most effective therapies. The quality initiative nature of the site should grant protective disclosure.

Table 2: Evolution of Ethical and Regulatory Framework

370 BC: Hippocratic Oath that specified a prime duty of a physician - to avoid harming the patient.

1947: Nuremberg Code highlighted the essentiality of voluntariness of this consent.

1948: Universal Declaration of Human Rights expressed concern about rights of human beings being subjected to involuntary maltreatment.

1962: Kefauver-Harris amendments manufacturer provides proof of the effectiveness and safety of their drugs before approval.

1964: Helsinki Declaration outlines general principles and specific guidelines on use of human subjects in medical research.

1974: US National Research Act and 1979: Belmont Report were major efforts in shaping ethics of human experimentation a result of Henry Beecher's 1966 study of abuses and the discovery of human exploitation of Tuskegee study

1996: International Conference on Harmonization, Good Clinical Practice, is the universal standard for ethical conduct of clinical trials.
The protocols that are approved should be "model" based. Medicine has been an "observational" science. When there is no knowledge, observation is the only source of new information. It is an inefficient process. Registries promote observational medicine. Other sciences have models that are tested until the model is accepted, refined, or rejected. The model can then be used to make predictions of future interventions. Medical research has accumulated enough observation to become model driven. Model-based therapies will also allow for multiple interventions which currently are not allowed in clinical trials. Traditional observational medicine changes one thing, keeping all other parameters the same, and observes the outcome. Changing a single parameter makes for easy statistical review. However, there is no one intervention, or magic bullet that will change a complex biologic system like the immune system. It takes multiple interventions to reset the immune system. Protocols that are submitted will be evaluated for the biologic model they represent; multiple interventions will be allowed. Open source allows multiple models to be tested. A successful protocol suggests the model of disease is correct.

\section{Method - Components of Open Research}

A web site that is accessible to all registered physicians. Open source means access to all. The data is collected under protective disclosure for a quality initiative. Acknowledgement of adverse and beneficial therapies is necessary for the physician to pick a protocol and for the results to find the most beneficial protocol in these diseases that are difficult to study. Each physician will serve as a safety committee and decide the appropriate protocol. This iterative method should identify patients that benefit from the protocol so that more of these patients are selected for success. A biostatistician will update results so a summary of information is also available to the physician. The site will need to validate that the users are registered physicians. On this site will be educational forums containing downloadable publications, (video educational forums explaining the model of disease and therapeutic rationale). The protocol, consent forms are accessible for downloading in multiple languages. The site will provide data entry in a depersonalized form with codes that can be accessed only by the computer for follow-up anonymous questionnaires of patients who have given consent for follow-up. Images will be downloaded, so raw data is available for review and not just an interpretation. This will allow core interpretation for confirming disease extent and response to therapy.

Governing Body Personnel will be selected by professional societies directly impacted by the clinical problems the open resource research is trying to answer. For example, for peripartum myocardial infarction - world-wide professional societies of cardiology, ob/gyn, heart failure, immunology, and others will be invited. Their responsibilities will be to review and approve protocols for scientific merit and safety concerns, data monitoring for safety concerns, and provide periodic summary of data. All publications will be reviewed by this committee. After peer review of the publications, the journal articles will then be posted on the site as online open access. Intellectual property will belong to the governing body and resources obtained from 
the intellectual property will be managed by this committee and distributed to worthy researchers. The governing body will be composed of selected respected researchers who will serve only as advisors. The voting body will be made up of two young investigators selected by each respected researchers. This youthful group will be the voting and regulatory arm. They should serve for three years and then be replaced.

Data Partner is an integral part of open access to assure security, accurate data collection and analytic review. The partner will have to have a global network of servers and redundancy of data to assure the input of data and data banks are accurate and to standards. Data collected will be demographic, laboratory results, protocol used, protocol deviations, medical images. The physicians who entered their patients will complete the data set. The case material with full de-identified clinical information will be immediately available to physicians deciding on a protocol and for expert adjudication.

Biostatistician This individual will be employed by the governing body to assure accuracy of clinical data and provide statistical review as data accumulates. Initially, results will be compared to historical controls and as more data is accumulated the individual protocols will be compared. His job will be similar to James Lind finding the protocols that have the best success.

Tissue, genetic, proteomic specimens will be collected with the appropriate consent of the patients for analysis. The specimens will be released by the governing committee to laboratories across the world that have applied for specimens and have demonstrated expertise. All results will be open and shared with the entire community of researchers. Intellectual property will belong to the group and not the individual. Open forum between researchers around the world will be provided by the data manager. Transparency at all levels is necessary. The goal is to solve problems and not obtain research grants, fame, or fortune. Reward the group and not the individual.

Funding of the governing body will be sponsored by professional societies since the goal of disease management is the same goal as professional societies. The web site could be sponsored by major medical journals or government. The data partner would be the industry that manages the "Cloud", approached as an altruistic motive. Other incentives to the data partner are this new innovative platform can be applied to other disciplines as well. Therapies provided will be at the expense of the local health care system. The advantage to them is a timely solution that can save future dollars in these rare, but expensive medical problems. The income from intellectual property could be used to fund future research independent of government funding eventually self-funding its research efforts. Contractual agreements with governments and industry will be allowed as long as these agreements meet the needs of disease management and are approved by the governing committee. Funds will be distributed by the same organization to researchers who do the work.

Physician Prospective - Rare diseases like peripartum myocardial infarction, peripartum cardiomyopathy, and myocarditis have no clinical treatment guidelines. They are difficult to study. When confronted with an unusual disease process, it is frustrating to watch patients suffer. Open source research provides to the physician treatment protocols based on biologic models. They can select the therapy that they feel best represents the biological model. Treating the patient and entering the data will soon give evidence that the treatment was successful. Physicians will be more likely to enroll patients when they have active control of the therapies. Protocol violations can also be studied that may lead to other knowledge that can affect the model of disease.

Researcher Prospective - The researcher can have access to difficult to obtain biological samples. Networking with other researchers can solve problems at a faster rate by notonly reducing duplications, but by also allowing confirmation of results. The researcher will have to give up the individual glory that has been the incentive and the disincentive of these individuals as they apply their knowledge. The model of rewarding the individual will be replaced by rewards to the group. The researcher will have to shift ideas of success from individual to group. The openness of this model can simplify applications and replace lengthy grant writing. Chat rooms provided by the site open to registered researchers can be an intellectual boom.

\section{Specific Aims - Application of Open source to Peripartum Myocardial infarction}

For example, in one year, 450 cases of postpartum myocardial infarction can be collected in the United States alone [14]. To really understand the disease cases from the entire world need to be collected. Coronary dissection, thrombosis, and spasm have all been implicated in peripartum myocardial infarction. There is overlap of these three conditions and all can coexists within the same patient. The current method of stent deployment, surgery, or medical therapy for coronary dissection is controversial. There are adverse reports of extending the dissection to other vessels making an infarct more extensive and reports of complete healing of the vessel without intervention. Current protocols utilize angioplasty, surgery or medical therapy in the treatment of peripartum myocardial infarction.

We have reported two case reports, figure 1, using the protocol listed in table 3 that only intervened on the immune and thrombotic pathways. These cases occurred 7 years apart; both, had multi-vessel infarctions and had dramatic improvement in their outcome with no residual cardiac disease. There was evidence in both of these cases of thrombosis and dissection and spasm events. The biologic model behind these two rare events is proposed as fetal paternal antigens stimulating an abnormal immune response to maternal coronary endothelium. ${ }^{6}$ The protocol uses multiple interventions to reset the immune system. It appears to be safe since it is immune modifying and not immune suppressive. It can provide sera from the plasmapheresis which may be analyzed by research groups to identify the abnormal immune pathways and abnormal antibodies. Our proposed method to treat the underlying ivmmune process is appealing when the coronary anatomy cannot be re-vascularized. This method is listed in table 3 . 
The author realizes that interventional doctors will be evaluating these patients and the bias will be to perform intervention. See Figure 2, 3 for the interventional decision and treatment pathways. There are 11 potential groups that can be treated initially and bail out procedures performed if initial success is not achieved.

The interventionist could choose to intervene in the discrete groups 2 through 7 . In these groups the specific aim (1) would be to determine if intervention is beneficial or harmful and characterize the angiographic characteristics of each. Flow in the vessel, additional complications such as closure of a new vessel because of dissection would be one metric, death or need for bailout procedure is another metric along with current echo and follow up echo in 6 months. The hypothesis (1) is intervention and surgery is harmful in these groups.

In groups 8 through 11 the diffuse nature of the disease could be treated by multiple vessel angioplasties, surgery, standard medication, or treated by the immune modifying protocol. In these groups outcomes can be compared. The outcomes would be death, left ventricular function during hospitalization and at 6 months. In the cases that were treated with angioplasty similar angiographic scores will be evaluated. The specific aim (2) would be determine if immune modification is better than traditional angioplasty, surgery, or medications. The Hypothesis (2) peripartum myocardial infarction is an immune disease and immune modification can favorably alter the outcome.

The open source model displaying outcomes of each treatment for physician review will iterate to the most successful therapies. The final answer may be immune therapy for all cases or will describe cases that are best suited for catheter intervention. The open source model will determine if the immune hypothesis is correct and how effective is immune modification. Open source means that new protocols could be introduced based on new information. The protocols can be adjusted and eventually the
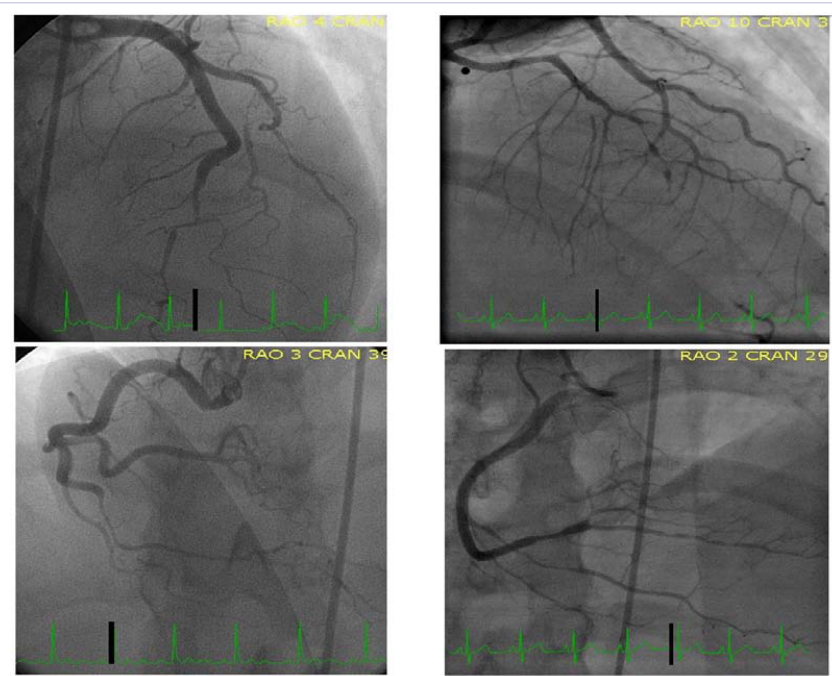

Figure 1: Two Cases of Peripartum Myocardial Infarction Sucessfully Treated with Immune Modifying Protocol Left and Right Coronary Artery
Table 3: Protocol for Peripartum Immune Related Disorders

\section{Plasmapheresis}

Plasmapheresis should be initiated as soon as possible and repeated early in the admission. The intended therapy is to rapidly address a humeral immune mediated process and to favorably alter rheological and thrombotic properties of blood. In addition, Intravenous Immune Globulin (IVIG) should be given after the first plasmapheresis is completed and repeated after exchanges or during a holiday from plasma exchange. In both cases, plasma exchange sequence was guided by response to therapy. Concern for bleeding risk was judged by fibrinogen value or by clinical impression of postpartum blood loss. Either albumin or fresh frozen plasma may be used for volume replacement. Daily clotting factors and fibrinogen levels should be obtained to help make this decision.

IVIG

60 GM IV after completion of plasma exchange times 4. Pretreatment with Acetaminophen $500 \mathrm{mg}$ and Benadryl 50mg may be given. Infusion rate 0.5 $\mathrm{ml} / \mathrm{kg} / \mathrm{hr}$ for 30 minutes increased to $1 \mathrm{ml} / \mathrm{kg} / \mathrm{hr}$ for 30 minutes, then $2 \mathrm{ml} /$ $\mathrm{kg} / \mathrm{hr}$. Do not give prior to a plasma exchange.

\section{Steroids}

\section{Solumedrol 1gm every day times 3}

Then begin oral prednisone 50mg daily with every other day taper of $10 \mathrm{mg}$ until $10 \mathrm{mg}$ per day is achieved. This represents a burst for approximately two weeks. Continue $10 \mathrm{mg}$ per day for three weeks, then reduce to $5 \mathrm{mg}$ for one week, then $5 \mathrm{mg}$ every other day for a week, then $2.5 \mathrm{mg}$ every other day for an additional week, then stop. Increase dose for flares in disease activity

\section{Anti-Platelets}

Aspirin $324 \mathrm{mg}$ initially, on discharge reduced to $81 \mathrm{mg}$ for life

Clopidogrel $300 \mathrm{mg}$ load on presentation, $75 \mathrm{mg}$ daily for 6 months, discontinued after favorable performance on treadmill

\section{Anti-Thrombin anticoagulation}

Heparin bolus and infusion per protocol (plasmapheresis line placed with imaging while on heparin by experienced personnel)

Warfarin therapeutic INR before heparin is discontinued and continued for 3 months

\section{Anti-Ischemic}

IV nitroglycerine transitioned to oral nitrates

Calcium channel blockers if ischemia on nitrates

Post Myocardial infarction (anti-inflammatory)

Beta blockers if tolerated initially and continued for life

ACEI for 6 months, consider discontinuing if left ventricular function is normal and blood pressure is normal

Statin on admission at least 6 months consider discontinuing if Hs-CRP low Spironolactone if ejection fraction is reduced.

Heart Failure (anti-inflammatory)

Beta blockers if tolerated initially and continued for life

ACEI for 6 months, consider discontinuing if left ventricular function is normal and blood pressure is normal

Statin on admission at least 6 months, consider discontinuing if Hs-CRP is low Spironolactone

Other congestive heart failure therapies as needed including balloon pump, loop diurectics, Nesseritide, RVAD, LVAD, and Defibrillators including biventricular devices

\section{Lactation}

Lactation by breast pump is potentially beneficial; however, breast feeding of the infant should be avoided if maternal medications have an adverse effect on the infant.

\section{Birth Control}

Avoid birth control pills first 6 months, abstinence, barrier method and preferred method is vasectomy of partner. Advise against future pregnancies. IVIG - Intravenous Immunoglobulin; ACEI - Angiotensin Converting Enzyme Inhibitor; Hs-CRP - High-Sensitivity C - reactive protein; RVAD - Right Ventricular Assist Device; LVAD - Left Ventricular Assist Device. 

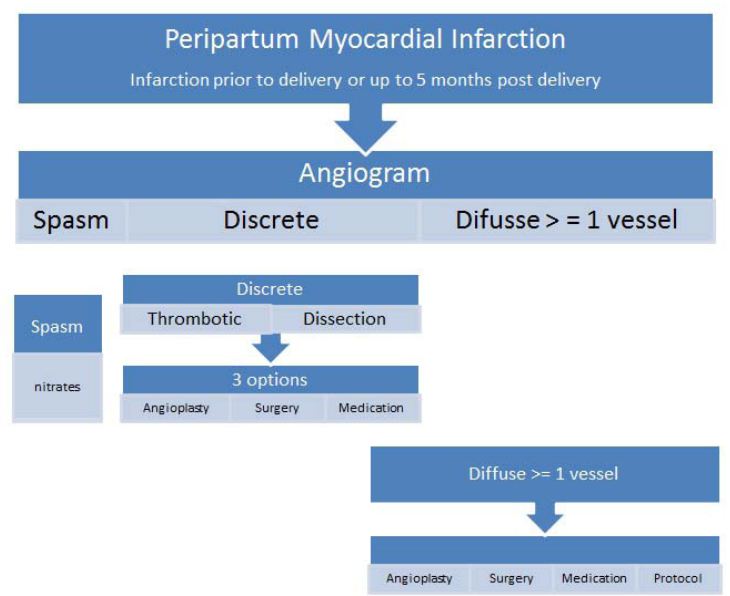

Figure 2: Possible Treatments

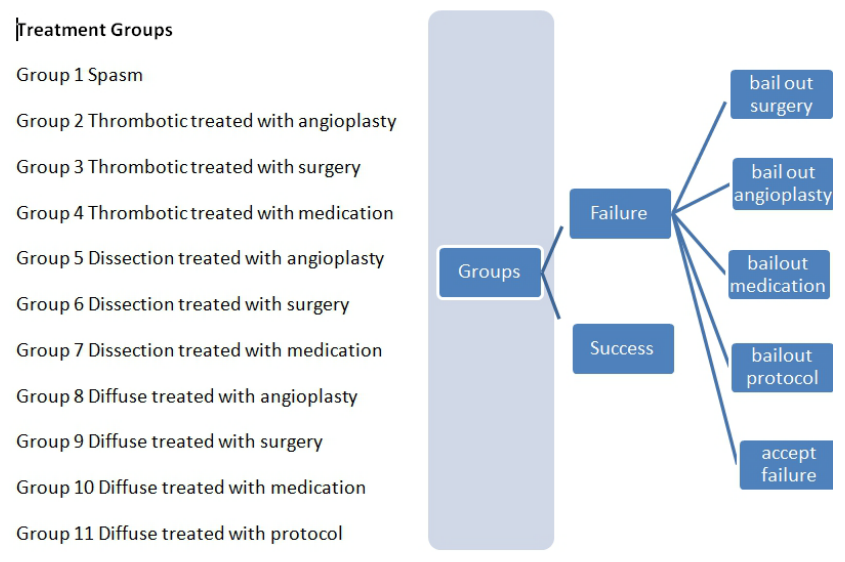

Figure 3: Treatment Groups

most optimum therapy is elicited and, at the same time, a biologic model of a rare disease is confirmed. Peripartum cardiomyopathy, myocarditis, and other difficult to study diseases could be managed by this same method. It is not a simple registry but a protocol driven registry to answer difficult clinical questions.

The major advantages of open source method:

1. Professional governing body reviews protocols for safety and biologic model.

2. The Doctor may select the protocol for his patient

3. The results are made available in real time to help select the protocol for the next case serving as continuous safety monitoring. Summaries presented by biostatistician.

4. Local IRB's are sent protocols by the governing body for their approval instead of investigator-initiated protocol. The protocols are approved prior to a rare case presenting. The protocol, consent forms are immediately available without delay being available online.

5. This method fosters group participation and rewards moving away from the individual centered research promoting disclosure and cooperation.

6. Funding is minimal with the cooperation of professional societies, treatment costs being absorbed by local health systems.

7. The web site becomes the definitive source of information of medical literature on those rare diseases; chat room on the site will allow collaboration of researcher working on a common problem.

8. This model can be applied to other rare diseases

\section{Conclusions}

Open source research is a step back in time to the method of James Lind and the simple controlled clinical trial. This method was successful in proving the nutritional model of Scurvy. This method when applied to rare diseases can have success confirming models of disease and therapy. It presents an option to treat and study rare disorders. Open source research is clearly different from registries where the results are only recorded. Open source utilizes biologic models that determine protocol selection. The physician is educated in these models and makes a decision as to what protocol to choose. The author's hypothesis is that peripartum myocardial infarction is an immune mediated disease that should be treated with immune modifying therapies. Open source research is a method to prove this hypothesis. Open source would allow another biologic model to solve this problem.

\section{References}

1. Roth A, Elkayam U. Acute myocardial infarction associated with pregnancy. Annals of Internal Medince 1996;125(9):751-762.

2. Roth A, Elkayam U. Acute Myocardial Infarction Associated With Pregnancy. Journal of the American College of Cardiology. 2008;52(3):171-180. doi: 10.1016/j.jacc.2008.03.049

3. The California Pregnancy-Associated Mortality Review (CA-PAMR). 2011. Available from: http://www.cdph.ca.gov/data/statistics/ Pages/CaliforniaPregnancy-AssociatedMortalityReview.aspx

4. Elkayam U. Clinical characteristics of peripartum cardiomyopathy in the United States: diagnosis, prognosis, and managment. J Am Coll Cardiol. 2011;58(7):659-670. doi: 10.1016/j.jacc.2011.03.047.

5. Houck PD, Linz W. Multi-Vessel myocardial infarction a window to future treatments of myocardial infarction. Heart Asia. 2010;2(1):8288. doi: 10.1136/ha.2009.001545.

6. Houck PD, Strimel WJ, Gantt DS, Linz WJ. Should we establish a new protocol for the treatment of peripartum myocardial infarction? Texas Heart Institute Journal 2012;39(2):244-248.

7. Pearson GD, Veille JC, Rahimtoola S, Hsia J, Oakley CM, Hosenpud JD, et al. Peripartum Cardiomyopathy - National Heart, Lung, and Blood Institute and Office of Rare Diseases (National Institutes of Health) Workshop Recommendations and Review. Journal of the American Medical Association. 2000;283(9):1183-1188.

8. Bhatt A. Evolution of clinical research: a history before and beyond James Lind. Perspectives in Clinical Research. 2010;1(1):6-10.

9. James Lind: The Visionary behind Modern Clinical Trials. Available from: http://www.jli.edu.in/blog/james-lind-the-visionary-behind- 
modern-clinical-trials/http://www.jli.edu.in/blog/james-lind-thevisionary-behind-modern-clinical-trials/

10. Hill AB. Suspended judgment: Memories of the British Streptomycin Trial in Tuberculosis. The first randomized clinical trial. Controlled Clinical Trials. 1990:11(2);77-79.

11. Perlman, D. Ethics in clinical research: a history of human subject protections and practical implementation of ethical standards. SoCRA SOURCE. 2004. Available from: http://materiais.dbio.uevora.pt/MA/ Modulo2/Artigos/SoCRA-Perlman.pdf
12. Nolan JP, Morley PT, Vanden Hoek TL, et al. Therapeutic Hypothermia After Cardiac Arrest. An Advisory Statement by the Advanced Life Support Task Force of the International Liaison Committee on Resuscitation Circulation. 2003;108:118-21.

13.Open-source movement. Available from: https://en.wikipedia.org/ wiki/Open-source_movement

14. Ladner HE, Danielson B, Gilbert WM. Acute myocardial infarction in pregnancy and the puerperium: a population-based study. Obstetrics and Gynecology. 2005;105(3):480-484. 